Iraqi Journal of Information and Communications Technology(IJICT)

Conference Series: The $1^{\text {st }}$ Conference of Applied Researches

in Information Engineering(ARIE2021), 2021

ISSN:2222-758X

e-ISSN:2789-7362

\title{
IMPROVED ROUTING PROTOCOLS Based ON RPL FOR Full IOT-WSN STACK
}

\author{
Sarah A. Rafea ${ }^{1}$, Abdulkareem A. Kadhim ${ }^{2}$ \\ 1,2 College of Information Engineering, Al-Nahrain University, Baghdad, Iraq \\ \{sara.ammar, abdulkareem.a \} @ coie-nahrain.edu.iq ${ }^{1,2}$ \\ Received:4/5/2021, Accepted:28/5/2021
}

\begin{abstract}
The Internet Engineering Task Force (IETF) standardized several protocols such as Constraint Application Protocol (CoAP) to run over WSN-IoT constrained devices. IPv6 is used to transmit packets over an IEEE802.15.4 radio link called 6LoWPAN. The routing protocol for low power and lossy (RPL) network enable connectivity of WSN over IoT. Nodes in RPL optimized their path using the objective function (OF), which depends on different node/link metrics. In this paper, the performance of the IoT-WSN stack consisting of CoAP, 6LoWPAN and two proposed protocols based on RPL are evaluated. The two proposed protocols depend on a new OF based on link reliability and energy metric. The first is a modification of a recently introduced protocol called Energy Threshold RPL (ETRPL) protocol. ETRPL uses the remaining energy of the preferred parent node as a metric. The other protocol used a new metric that combines energy consumption with ETX for all paths to the root called EERPL. The protocols are tested with a full IoT-WSN stack and implemented using the Cooja simulator. The results showed that ETRPL and EERPL performed better than standard RPL in terms of energy consumption, average time delay, packet reception ratio, throughput and the number of dead nodes.
\end{abstract}

keywords: Routing protocols, Wireless sensor networks, Internet of things, RPL, CoAP, Objective function, Cooja.

\section{INTRODUCTION}

Internet of Things (IoT) is a technology that enables intelligent things to collect and exchange data through the internet. Things refer to any physical object with a device that has its IP address and can connect to a network to send/receive data via the network. According to CISCO estimation, the number of connected devices with each other through IoT has increased to about 50 billion devices by 2020 [1]. These huge numbers of devices need to be connected by an efficient protocol stack to guarantee a prolonged lifetime of the IoT network. Wireless Sensor Networks (WSNs) distribute hundreds to thousands of inexpensive micro-sensor nodes in their areas [2], and these nodes are fundamental parts of IoT. There are three main approaches to connect WSN to the Internet depending on the integration degree of the Internet structure [3]. In the first approach, the WSN is connected to the Internet through a single gateway causing a single point of failure. In the second approach, few nodes can access the Internet constricting a hybrid network. In the third approach, several nodes can access the Internet in a single hop. All the mentioned three approaches are inflexible because they support only static network configuration and each time there is a need to add devices thus the gateway must be reprogrammed and hence causing a delay. To achieve flexibility, the node in WSN must work with an IP address to communicate with each other through the Internet. In this case, the gateway function will be limited to forward data and to translate between the protocols. IETF specifies an adaptation layer to enable IPv6 carried over IEEE 802.15.4 radio link, this adaptation layer is called IPv6 and is based on LoW Personal Area Network (6LoWPAN) [3]. In 2010, the Constrained Restful Environments (CoRE) working group created the Constrained Application Protocol (CoAP) which defines a mechanism to communicate a large number of small devices, low power, and resource-constrained devices over lossy networks [4], [5]. CoAP is a protocol that works at the application level as a web transfer protocol for use with constrained nodes and constrained networks [6]. In March 
Iraqi Journal of Information and Communications Technology(IJICT)

Conference Series: The $1^{\text {st }}$ Conference of Applied Researches

in Information Engineering(ARIE2021), 2021

ISSN:2222-758X

e-ISSN:2789-7362

2012, IETF standardized a routing protocol to implement IoT over WSN called routing protocol for low power and lossy network (RPL) [7]. RPL is a proactive protocol based on IPv6. RPL is designed to meet the requirements of a wide range of applications such as smart city applications, industrial applications, medical applications,...etc [8]. Many application layer protocols investigated in [9], the CoAP protocol is the most lightweight and consume low energy. In [10], the performance of application protocols, namely Message Queuing Telemetry Transport (MQTT), CoAP, and others are investigated and compared. Three parameters are measured: bandwidth, latency and packet loss. The results showed that CoAP is potentially used for certain applications that require low bandwidth consumption and low latency. The application of monitoring systems for an indoor environment is implemented in [11] using the Cooja simulator to simulate the sensor node. Seven nodes are deployed in a $20 \times 20 \mathrm{~m} 2$ area for collecting data. The performance of the IoT-WSN protocol was investigated in [12] using the Cooja simulator and Californium [13] framework. Five nodes are deployed as temperature and humidity sensors and one node as a border router. A comprehensive study using the Cooja simulator in Contiki operating system covered the effects of the two objective functions used in RPL (the Minimum Rank with Hysteresis Objective Function (MRHOF) and Objective Function Zero (OF0)) on the network performance. Different network topologies, number of nodes and transmission ranges were considered. The results showed that OF0 usually performed better than MRHOF in terms of energy consumption, listening duty cycle, and transmit duty cycle [14] . Expected Transmission Count (ETX), which is the number of retransmissions required over a given path, and Received Signal Strength Indicator (RSSI) is used to enhance the performance of RPL protocol [15], where an algorithm is suggested to control the transmission power of a given node concerning distance. The algorithm is also tested using the Cooja simulator with two nodes. Different objective functions, such as hop count, ETX and RSSI-ETX in large scale networks (from 200 to 600 nodes) are investigated. As a result, it is found that the mean end to end delay is decreased when using the hop count objective function with a greater packet delivery ratio when using the RSSI-ETX objective function using OMNET++ simulator [16] . In 2018, many researchers used energy as a parameter metric together with other metrics. Stability study for RPL is presented using two different objective functions; the link metric MRHOF-ETX and node metric MRHOF-Energy in Cooja simulator with 30 nodes [17]. The results showed that the energy consumption is decreased by about $28 \%$ when using MRHOF-Energy objective function, but the stability of MRHOF-Energy is lower than that of MRHOF-ETX by about 1.3\% [17]. In 2019, Energy Threshold based on RPL (ETRPL) is proposed to prolong the lifetime of the network. ETRPL depends on ETX and energy metrics to select the optimal path to the root. ETRPL works in a way that each node changes its parent if the energy of its parent became below a certain energy threshold. Different energy thresholds are investigated with a different number of nodes and network coverage areas. ETRPL was also implemented using the Cooja simulator. The results showed that the ETRPL protocol is useful for IoT networks with relatively small areas [18] . In the present paper, RPL based on energy and ETX (EERPL) protocol is proposed to improve the energy consumption of the network and hence prolong the lifetime of the network. EERPL and its variant ETRPL protocols combine ETX metric as a link metric with energy metric as node metric in two different ways to define the proposed objective function. CoAP protocol is also used as an application protocol at the top of ETRPL and EERPL protocols. The rest of this paper is organized as follows: Section II presents the IoT-WSN protocol stack in detail. In Section III, the proposed objective function is introduced. The performance evaluation 
Iraqi Journal of Information and Communications Technology(IJICT)

Conference Series: The $1^{\text {st }}$ Conference of Applied Researches in Information Engineering(ARIE2021), 2021

ISSN:2222-758X e-ISSN:2789-7362

of RPL, ETRPL, and EERPL protocols is presented in Section IV. Finally, the conclusion is presented in Section V.

\section{IOT-WSN PRotocol Stack}

Many protocols have been standardized to facilitate the functionality of IoT. IoT protocol stack is designed to work on resource-constrained devices rather than the traditional web stack [19]. The comparison between IoT stack and Web stack is presented in Fig. 1. The IoT-WSN stack is a special case of IoT stack. Since the node in WSN is resource-constrained device, it needs to be operated efficiently in all stack layers. The IoT-WSN protocol stack is presented in Fig. 2 [20].

\begin{tabular}{|c|c|c|c|}
\hline \multirow[b]{2}{*}{ TCP/IP Model } & \multicolumn{2}{|c|}{ IoT Stack } & \multirow{2}{*}{$\begin{array}{c}\text { Web Stack } \\
\text { Web Application }\end{array}$} \\
\hline & $\begin{array}{c}\text { IoT } \\
\text { Applications }\end{array}$ & $\begin{array}{c}\text { Device } \\
\text { Management }\end{array}$ & \\
\hline Data Format & \multicolumn{2}{|c|}{ Binary, JSON, CBOR } & HTML, XML, JSON \\
\hline Application Layer & \multicolumn{2}{|c|}{ CoAP, MQTT, XMPP, AMQP } & HTTP, DHCP, DNS, TLS/SSL \\
\hline Transport Layer & \multicolumn{2}{|c|}{ UDP, DTLS } & TCP, UDP \\
\hline \multirow{2}{*}{ Network Layer } & \multicolumn{2}{|c|}{ IPv6/IP Routing } & \multirow{2}{*}{ IPv6, IPv6, IPSec } \\
\hline & 6Lo & PAN & \\
\hline \multirow{2}{*}{$\begin{array}{l}\text { Link Layer } \\
\text { Physical Layer }\end{array}$} & \multicolumn{2}{|c|}{ IEEE 802.15.4 MAC } & \multirow{2}{*}{$\begin{array}{c}\text { Ethernet (IEEE 802.3), DSL, } \\
\text { ISDN, Wireless LAN (IEEE } \\
802.11 \text { ), Wi-Fi }\end{array}$} \\
\hline & \multicolumn{2}{|c|}{ IEEE 802.15.4 PHY } & \\
\hline
\end{tabular}

Figure 1: IoT and web stack [19]

\section{CoAP}

\section{UDP/ICMPv6}

\section{IPv6 and RPL}

\section{LOWPAN}

\section{IEEE 802.15.4 MAC}

\section{IEEE 802.15.4 PHY}

Figure 2: IoT-WSN protocol stack [20] 


\section{A. Constrained Application Protocol}

The CoAP protocol is based on REST and its algorithm is similar to HTTP by exchanging data between clients and servers. The work of CoAP is based on the fact that one thing (CoAP client) can send a request to another thing (CoAP server) as shown in Fig. 3 [5], [21]. CoAP used four types of messages: confirmable (CON), non-confirmable (NON), acknowledgement (ACK), and reset (RST). In CON message the response that must be sent is either positive or negative acknowledgement. In case if there is no acknowledgement, the node must keep exponentially retransmitting messages until an acknowledgement is received. NON-messages are used in an unreliable transmission such as a temperature measurement being measured periodically so the missing of one measurement is not considered as a big problem. This message does not need an acknowledgment. ACK message is sent as an acknowledgment message to the CON message. RES message represents a negative acknowledgment and it refers to a failure in general [5].

\section{B. IPv6 Based on the Low Personal Area Network}

6LoWPAN is considered as an adaptation layer that fits IPv6 packets with IEEE 802.15.4 specifications [22]. The 6LoWPAN specifications support different length addresses, different topologies, low bandwidth, low cost, low power consumption, scalable networks, mobility and long sleep time [23]. 6LoWPAN provides header compression and fragmentation mechanism and supports multi-hop delivery. 6LoWPAN used four types of headers to envelop the datagram: "NO 6LoWPAN Header", "Dispatch Header", "Mesh Addressing" , and "Fragmentation" . The packets that are not compressed according to 6LoWPAN specifications will be discarded by "NO 6LoWPAN Header". "Dispatch Header" is responsible for multicasting or compression of the IPv6 header. "Mesh Addressing Header" identifies the packets that have to be forwarded to the link layer. "Fragmentation Header" is used in the case where datagrams length is larger than a single IEEE802.15.4 frame. 6LoWPAN can compress IPv6 headers to two bytes [21].

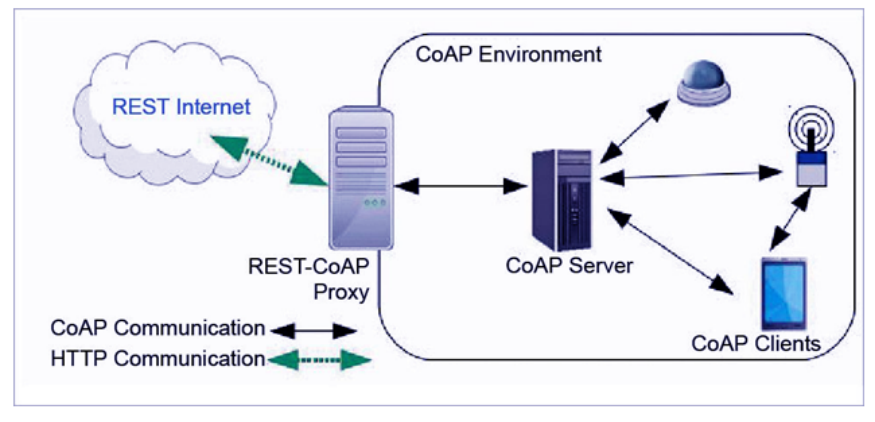

Figure 3: CoAP functionality [21]

\section{Routing Protocol for RPL Network}

The routing protocol for the RPL network is a tree-like topology called Destination-Oriented Directed Acyclic Graphs (DODAG), which is Directed Acyclic Graphs (DAG) with one destination root as shown in Fig. 4 [24]. This formation of network generate loops, so ranking is used to define the position of the node relative to other nodes concerning a DODAG 
root [24]. RPL nodes can be root, router or leaf nodes. The root node is a special node programmed to be the destination of all other nodes and considered as a border router. The router node forwards data packets from its child to the root. Leaf node on the other hand just sends its data to preferred parents [7], [9].
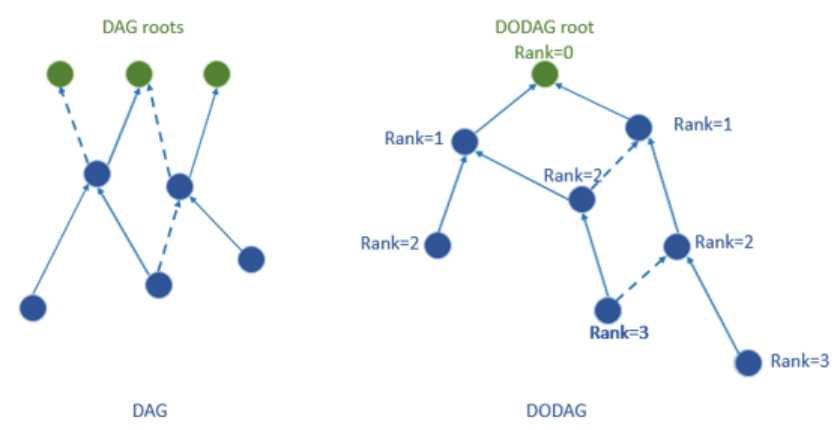

Figure 4: RPL DAG and DODAG

\section{1) RPL Control Messages}

RPL used four types of control messages which are [21], [24] :

- DODAG Information Object (DIO) that contains routing metric and OF to be advertised to all nodes to select a DODAG parent.

- DODAG Information Solicitation (DIS) is used by any isolated node that wants to join DODAG when no DIO message is received.

- Destination Advertisement Object (DAO) send by a child as a request to join a DODAG.

- Destination Advertisement Object (DAO-ACK) send as an acknowledgement to the DAO message.

RPL used upward routing and downward routing to build its topology. The node used the DIO message to construct its path upward to the root and DAO message in downward routing.

2) RPL Topology Construction

The topology construction of the RPL network starts when the root multicast the DIO message. Each node that receives a DIO message from the root will set the root as a preferred parent, calculate rank, calculate path cost, and send a DAO message to the root. Each node then multicasts a DIO message with the new parameter. Each node used an objective function (OF) to calculate its rank and path cost to root to select its preferred parent.

3) RPL Objective Function

RPL selects an optimal route, construct DODAG topology and calculates the rank of each node based on OF [25]. OF used node metric and/or link metric to determine the optimal parent to the node and to calculate the rank value. Two objective functions are already used; OF0 [26] and MRHOF [25]. OF0 used hop count as a metric to determine the best path which is the nearest path to the root. MRHOF use ETX to compute the best path to the root. RPL can use other metrics such as energy, delay and received signal strength indicator (RSSI) [27]. 
Iraqi Journal of Information and Communications Technology(IJICT)

Conference Series: The $1^{\text {st }}$ Conference of Applied Researches

in Information Engineering(ARIE2021), 2021

ISSN:2222-758X

e-ISSN:2789-7362

\section{Energy Threshold RPL Protocol}

Energy Threshold RPL (ETRPL) protocol using a multicasting DIO message from the root to construct its topology as in RPL. Each node that receives the DIO message from the root will select the root as a preferred parent then multicast the DIO message with a new parameter to its neighbours. The parameter multicasting in the DIO message includes the new rank, the remaining energy, and other parameters. The receiver node checked the remaining energy received from each DIO input message. If the remaining energy of the sender is less or equal energy threshold, the node will increase the cost of its path to this sender to exclude this sender from being the preferred parent. ETRPL improve the energy consumption of the RPL network by implementing a new objective function. The new objective function uses two metrics; the first one is the ETX metric considered as a link metric and the other one is the energy metric considered as a node metric. ETRPL used ETX to calculate the path cost of a node and at the same time, it discards the parent with remaining energy less or equal to the energy threshold. In ETRPL, the node selects the best link quality at the same time switch parent if the remaining energy of parent became less or equal to the energy threshold also never chooses the parent with remaining energy already less or equal to the energy threshold. More details are found in [18].

\section{RPL BASED ON ENERGY AND ETX METRIC}

EERLP proposed a new metric called EE metric that combined the ETX metric and energy metric. EERPL used energy consumption of all paths from the node to the root and also used ETX value for all paths. EERPL adds the energy consumption of all paths to the ETX value of all paths to the root. The node in EERPL selects the best link quality and energy consumption path. EERPL protocol built its topology as in RPL protocol. The sender multicast DIO message that contains the cost of its path to root to its neighbours. When the node receives a DIO message, it adds path cost to its cost and multicast a DIO message. The path cost sent by the sender consists of consumed energy and ETX value (EE metric). Fig. 5 shows the flowchart of EERPL topology construction. In EERPL each node adds its ETX link value, Energy consumption and ETX value of its parent, and sends it to node neighbors as a single variable. The node selects the minimum EE metric value as a parent node. EERPL is designed to balance the energy consumption and link quality metric hence prolonging the lifetime of the network. In EERPL each node selects the minimum energy consumption with the best quality link. The node switches its parent in case when the node receives a DIO message with EE metric less than the EE metric of its current preferred parent. DIO message contains the consumed energy and the ETX value (EE metric). The EE metric sent by root is 0 because the consumed energy is always 0 since the root is always powered and the ETX value of root is also 0. Each node that received the DIO message will add the EE metric received from its parent to the ETX value of the link between the sender and the received node in the parent selection process. The node, after choosing its preferred parent, will add its consumed energy to the EE metric and send the new EE metric with a DIO message. The EE metric received in the DIO message contains the ETX value and the consumed energy of the path from the sender of the DIO message to the root. The parent selection process doesn't depend on the energy consumption of the same node. The node in EERPL selects the neighbour as the preferred parent with the best combination of ETX value and energy consumption to the root.

https://ijict.edu.iq 


\section{Simulation TeSts AND Results}

There are several real-time operating systems used to provide the functionality of IoT applications such as Contiki OS, Tiny OS, and others [21] . Contiki OS is one of the most widely used operating systems for WSN supporting IoT. Cooja simulator is the tool in Contiki OS used to simulate and emulate WSN over IoT. It is mentioned that about $63 \%$ of all simulation studies used the Cooja simulator [28] . Contiki OS 3.0 is used to simulate the network for implementing and testing the given protocols. The protocol stack for the embedded operating system in Contiki is called uIPv6. The uIPv6 is an implementation of IPv6 with 6LoWPAN. Californium is a framework that implements CoAP in Java. It provides an API for RESTful Web services that support CoAP's features. The throughput of the Californium CoAP framework shows 33 to 64 times higher than high-performance HTTP Web servers [12] . The performance evaluation covered RPL, ETRPL and EERPL protocols. In the evaluation the following parameters are measured; Average remaining energy, Packet Reception Ratio (PRR), Average time delay, Throughput, and the number of dead nodes. Table I shows the main test parameters. The assumed topology deployed 20, 30 and 40 CoAP server nodes randomly in the given area. Two different areas are considered $100 \mathrm{~m} \times 100 \mathrm{~m}$ and $200 \mathrm{~m} \times 200 \mathrm{~m}$. CoAP client (Californium) requests resources from the CoAP server periodically through border router as in Fig. 6 . The resources can be temperature, humidity, ... etc. For the simulation purpose, the CoAP server will request resources represented by "Hello world" since the temperature and other resources cannot be simulated. The GET request will be used by the CoAP client. CoAP client requests different CoAP servers in every minute. The border router connects the CoAP server to the Internet through a tool in Contiki OS called Tunslip utility. In this research, the Tunslip connects the CoAP client with the CoAP server by creating a bridge through the local machine. For ETRPL, the energy threshold is selected to be $25 \%$ of the initial energy set for each node. Such threshold is considered as the best threshold according to related research [18]. Regardless of the exact value used for the initial energy, RPL, ETRPL, and EERPL protocols assumed that all nodes have $100 \%$ initial energy before starting any transmission.

TABLE I

The Main Parameters of The Tests

\begin{tabular}{|c|c|}
\hline Parameter & Specification or Value \\
\hline Topology & Random \\
\hline Number of nodes & 20,30 and 40 \\
\hline Simulation time & 60 Mins. \\
\hline Node type & Zolertia Z1 [29] \\
\hline Transmission range & $50 \mathrm{~m}$ \\
\hline
\end{tabular}

\section{A. The Average Remaining Energy}

The average remaining energy for the network is computed by the total remaining energies of all nodes divided by the number of nodes. The results in Tables II, III, and IV shows the average remaining energy for the two considered areas with 20, 30 and 40 nodes, respectively. The results show that ETRPL and EERPL consumed less energy compared to the RPL protocol since the ETRPL and EERPL protocol consider the energy metric. In a small area $(100 \times 100 \mathrm{~m} 2)$, the average remaining energy is increased as compared with the large area $(200 \times 200 \mathrm{~m} 2)$ for all numbers of nodes considered. A slight increase of the average remaining energy of ETRPL compared to the EERPL protocol is shown. Considering a 
Iraqi Journal of Information and Communications Technology(IJICT)

small area with a relatively high number of nodes; the average remaining energy is increased in ETRPL since there is a high number of nodes that can be selected as a parent node.

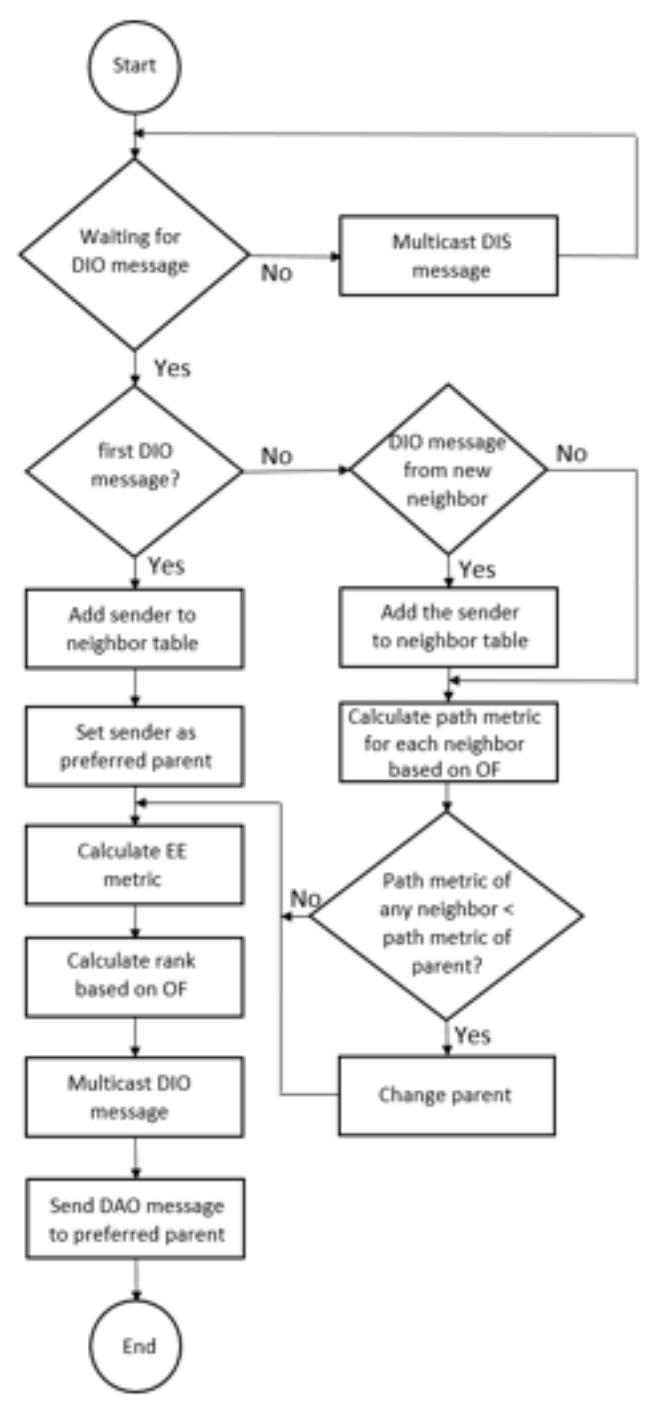

Figure 5: The flowchart of EERPL protocol 


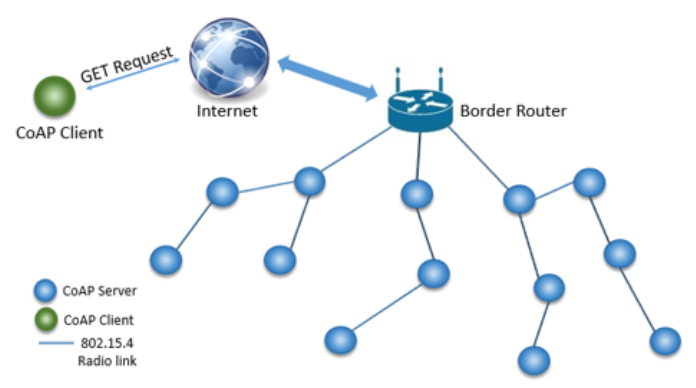

Figure 6: Simulation cenario

TABLE II

The Average Remaining Energy in mJ for 20 Nodes

\begin{tabular}{|c|c|c|c|}
\hline Area $\left(\mathrm{m}^{2}\right)$ & RPL & ETRPL & EERPL \\
\hline $100 \times 100$ & 424.94 & 1829.63 & 1760.21 \\
\hline $200 \times 200$ & 1221.1 & 1748.57 & 1568.47 \\
\hline
\end{tabular}

TABLE III

The Average Remaining Energy in mJ for 30 Nodes

\begin{tabular}{|c|c|c|c|}
\hline Area $\left(m^{2}\right)$ & RPL & ETRPL & EERPL \\
\hline $100 \times 100$ & 87.37 & 1857.03 & 1690.62 \\
\hline $200 \times 200$ & 615.27 & 1120.48 & 838.86 \\
\hline
\end{tabular}

TABLE IV

The Average Remaining Energy in $\mathrm{mJ}$ for 40 Nodes

\begin{tabular}{|c|c|c|c|}
\hline Area $\left(m^{2}\right)$ & RPL & ETRPL & EERPL \\
\hline $100 \times 100$ & 27.89 & 483.87 & 446.82 \\
\hline $200 \times 200$ & 429.10 & 982.61 & 936.3 \\
\hline
\end{tabular}

\section{B. The Packet Reception Ratio}

The packet reception ratio (PRR) is calculated by dividing the number of correct packets received by the CoAP client by the number of packets sent by the CoAP client. Tables V, VI, and VII show the PRR for 20, 30 and 40 nodes, respectively. In the small area, there is no loss in packets for the cases of 20 and 30 nodes for both ETRPL and EERPL protocols. For the case of a large area, the PRR is degraded but still higher than RPL. In general, for a small area, the PRR is better than that in a large area because, in a large area, the number of nodes ready to forwarded data is less than that in a small area, so there is always nodes ready to forward data in a small area.

TABLE V

The Packet Reception Ratio for 20 Nodes

\begin{tabular}{|c|c|c|c|}
\hline Area $\left(\mathrm{m}^{2}\right)$ & RPL & ETRPL & EERPL \\
\hline $100 \times 100$ & $98.95 \%$ & $100.0 \%$ & $100.0 \%$ \\
\hline $200 \times 200$ & $99.03 \%$ & $99.45 \%$ & $99.64 \%$ \\
\hline
\end{tabular}

TABLE VI

The Packet Reception Ratio for 30 Nodes

\begin{tabular}{|c|c|c|c|}
\hline Area $\left(\mathrm{m}^{2}\right)$ & RPL & ETRPL & EERPL \\
\hline $100 \times 100$ & $97.76 \%$ & $100.0 \%$ & $100.0 \%$ \\
\hline $200 \times 200$ & $96.74 \%$ & $98.99 \%$ & $98.22 \%$ \\
\hline
\end{tabular}


TABLE VII

The Packet Reception Ratio for 40 Nodes

\begin{tabular}{|c|c|c|c|}
\hline Area $\left(m^{2}\right)$ & RPL & ETRPL & EERPL \\
\hline $100 \times 100$ & $96.79 \%$ & $99.02 \%$ & $98.91 \%$ \\
\hline $200 \times 200$ & $98.32 \%$ & $96.89 \%$ & $99.2 \%$ \\
\hline
\end{tabular}

\section{The Average Delay Time}

The average delay time represents the time that the CoAP client takes to send a request until the response is received divided by the number of requests (measured in msec). The results in Tables VIII, IX, and X show the average delay time for two different areas with 20,30 and 40 nodes, respectively. The average delay time of ETRPL and EERPL is less than that of RPL in all cases. The average delay time of ETRPL is less than EERPL in all cases except for large areas with 40 nodes, because the EERPL mechanism for selecting parents depends on ETX and energy consumption. In many cases the path with the best energy consumption and link quality is longer than other paths, thus causing ETRPL to perform better than EERPL for most cases.

TABLE VIII

The Average Time Delay in msec. for 20 Nodes

\begin{tabular}{|c|c|c|c|}
\hline Area $\left(m^{2}\right)$ & RPL & ETRPL & EERPL \\
\hline $100 \times 100$ & 1315.75 & 404.73 & 446.12 \\
\hline $200 \times 200$ & 761.96 & 617.19 & 645.91 \\
\hline
\end{tabular}

TABLE IX

The Average Time Delay in msec. for 30 Nodes

\begin{tabular}{|c|c|c|c|}
\hline Area $\left(\mathrm{m}^{2}\right)$ & RPL & ETRPL & EERPL \\
\hline $100 \times 100$ & 3248.24 & 544.11 & 659.82 \\
\hline $200 \times 200$ & 2487.71 & 988.38 & 1155.51 \\
\hline
\end{tabular}

TABLE X

The Average Time Delay in msec. for 40 Nodes

\begin{tabular}{|c|c|c|c|}
\hline Area $\left(\mathrm{m}^{2}\right)$ & RPL & ETRPL & EERPL \\
\hline $100 \times 100$ & 4747.1 & 1378.67 & 1470.48 \\
\hline $200 \times 200$ & 2727.63 & 1452.96 & 1263.75 \\
\hline
\end{tabular}

\section{The Throughput}

The throughput of the network is calculated according to Eq. 1 [20]. The throughput is measured in bps.

$$
\text { Throughput }=(\text { No. of successfully request } *(\text { length of request }+ \text { length of response })) / \text { simulation time }
$$

Tables XI, XII, and XIII show the throughput for 20, 30 and 40 nodes, respectively, with the two different areas. The throughputs of ETRPL and EERPL are higher than that of RPL as expected. The throughput of EERPL is higher than that of ETRPL in all cases with a slight difference since the node in the ETRPL protocol changing its parent more frequently. Thus the node consumed time to change parent more than EERPL case causing the root to receive fewer packets and hence the throughput is degraded.

TABLE XI

The Throughput in bps for 20 Nodes

\begin{tabular}{|c|c|c|c|}
\hline Area $\left(\mathrm{m}^{2}\right)$ & RPL & ETRPL & EERPL \\
\hline $100 \times 100$ & 32.82 & 33.46 & 34.05 \\
\hline $200 \times 200$ & 27.49 & 29.28 & 30.29 \\
\hline
\end{tabular}


TABLE XII

The Throughput in bps for 30 Nodes

\begin{tabular}{|c|c|c|c|}
\hline Area $\left(\mathrm{m}^{2}\right)$ & RPL & ETRPL & EERPL \\
\hline $100 \times 100$ & 32.72 & 37.12 & 39.76 \\
\hline $200 \times 200$ & 22.16 & 28.93 & 26.56 \\
\hline
\end{tabular}

TABLE XIII

The Throughput in bps for 40 Nodes

\begin{tabular}{|c|c|c|c|}
\hline Area $\left(m^{2}\right)$ & RPL & ETRPL & EERPL \\
\hline $100 \times 100$ & 24.93 & 40.82 & 43.68 \\
\hline $200 \times 200$ & 25.12 & 23.33 & 30.02 \\
\hline
\end{tabular}

\section{E. The Number of Dead Nodes}

The node is considered a dead node when it consumes all of its energy. The dead nodes can't send and receive any message. Tables XIV, XV , and XVI show the throughput for 20, 30 and 40 nodes, respectively, with two different areas. There are no dead nodes in ETRPL and EERPL in a small area with 20 and 30 nodes compared to RPL. In a large area, the EERPL has a higher number of dead nodes than ETRPL with 20 and 30 nodes. For 40 nodes, ETRPL has a higher number of dead nodes compared to EERPL in small and large areas. The network with a high number of nodes consumes higher energy hence causing a high number of dead nodes.

TABLE XIV

The Number of Dead Nodes for 20 Nodes

\begin{tabular}{|c|c|c|c|}
\hline Area $\left(\mathrm{m}^{2}\right)$ & RPL & ETRPL & EERPL \\
\hline $100 \times 100$ & 8 & 0 & 0 \\
\hline $200 \times 200$ & 8 & 4 & 5 \\
\hline
\end{tabular}

TABLE XV

The Number of Dead Nodes for 30 Nodes

\begin{tabular}{|c|c|c|c|}
\hline Area $\left(m^{2}\right)$ & RPL & ETRPL & EERPL \\
\hline $100 \times 100$ & 19 & 0 & 0 \\
\hline $200 \times 200$ & 16 & 11 & 13 \\
\hline
\end{tabular}

TABLE XVI

The Number of Dead nodes for 40 Nodes

\begin{tabular}{|c|c|c|c|}
\hline Area $\left(m^{2}\right)$ & RPL & ETRPL & EERPL \\
\hline $100 \times 100$ & 26 & 12 & 11 \\
\hline $200 \times 200$ & 21 & 14 & 16 \\
\hline
\end{tabular}

\section{Conclusion}

The operation of a full IoT-WSN protocol stack including CoAP, 6LoWPAN and two enhancement protocols based on RPL are considered in this work. CoAP is an application protocol that works as a web transfer protocol used with the constrained device over a constrained network. 6LoWPAN is an adaptation layer used to enable IPv6 to be carried over IEEE 802.15.4 link. RPL is a routing protocol for resource constraint devices. The traditional RPL protocol selects its optimal path based on an objective function that depends either on ETX or hops count to select a preferred parent. The Energy Threshold RPL (ETRPL) and RPL based on energy and ETX protocol (EERPL) are proposed to improve the performance of the IoT-WSN protocol stack. ETRPL used OF based on remaining energy of preferred parent while EERPL 
Iraqi Journal of Information and Communications Technology(IJICT)

Conference Series: The $1^{\text {st }}$ Conference of Applied Researches

in Information Engineering(ARIE2021), 2021

ISSN:2222-758X

e-ISSN:2789-7362

used OF based on ETX and consumed energy. The full IoT-WSN protocol stack is tested using the Cooja simulator. The results show that the IoT-WSN protocol stack consumes less energy in the case of using ETRPL and EERPL protocol instead of the traditional RPL protocol. The highest average remaining energy was obtained in a small area with a small number of nodes and in a large area with a large number of nodes with a slite difference between ETRPL and EERPL. The IoT-WSN protocol stack also performs better with ETRPL and EERPL regards to PRR, average delay time, throughput and number of dead nodes with slite deference between ETRPL and EERPL.

\section{REFERENCES}

[1] D. Evans, "The Internet of Things - How the Next Evolution of the Internet is Changing Everything" , CISCO, San Jose, CA, USA, White Paper, 2011.

[2] A. A. Kadhim and M. W. Abdulrazzaq, "Efficient Routing Techniques for Wireless Sensor Networks", Journal of Applied Sciences, Vol. 14, No. 24. pp. 3479-3485, 2014.

[3] D. Christin, A. Reinhardt, P. Mogre, and R. Steinmetz, "Wireless Sensor Networks and the Internet of Things: Selected Challenges" , Proceedings of the 8th GI/ITG KuVS Fachgesprach Drahtlose sensornetze. pp. 31-34, 2009.

[4] I. M. Al-Joboury and E. H. Al-Hemiary, "Internet of Things (IoT): Readme" , 1st International Conf. Inf. Technol. , Vol. 2, No. 2, pp. 343-358, 2017.

[5] S. R. Jan, F. Khan, F. Ullah, N. Azim, and M. Tahir, "Using Coap Protocol for Resource Observation in Iot" , Vol. 21, No. 2, pp. 11-14, 2016.

[6] Z. Shelby, K. Hartke, and C. Bormann, "The Constrained Application Protocol (CoAP) " , pp. 1-112, 2014.

[7] T. Winter et al. , "RPL: IPv6 Routing Protocol for Low-Power and Lossy Networks", DOI 10.17487/ RFC6550, pp. 1-157, March, 2012.

[8] Z. Sheng, D. Tian, and V. Leung, "Towards Energy and Resource Efficient Internet of Things: A Design Principle Combining Computation, Communications and Protocols", IEEE Communications Magazine, Vol. 56, No. 7. pp. 89-95, July, 2018.

[9] A. Sebastian and S. Sivagurunathan, "A Survey on Load Balancing Schemes in RPL based Internet of Things" , IJSRNSC, Vol. 6, No. 3, pp. 43-49, 2018.

[10] Y. Chen and T. Kunz, "Performance Evaluation of IoT Protocols Under A Constrained Wireless Access Network" ,Int. Conf. Sel. Top. Mob. Wirel. Networking, MoWNeT, 2016.

[11] G. A. Costa and J. H. Kleinschmidt, "Implementation of a Wireless Sensor Network Using Standardized IoT Protocols", IEEE Int. Symp. Consum. Electron. Implement, pp. 1-2, 2016.

[12] S. Thombre, R. U1 Islam, K. Andersson, and M. Hossain, "Performance Analysis of An IP Based Protocol Stack for WSNs" , Comput. Commun. Work. (INFOCOM WKSHPS), IEEE Conf. , pp. 360-365, 2016.

[13] "Californium", https://www.eclipse.org/californium/.

[14] W. Mardini, M. Ebrahim, and M. Al-Rudaini, "Comprehensive Performance Analysis of RPL Objective Functions in IoT Networks" , International Journal of Communication Networks and Information Security, Vol. 9, No. 3. pp. 323-332, 2017.

[15] M. Mahmud, A. Abdelgawad, and K. Yelamarthi, "Energy Efficient Routing for Internet of Things (IoT) Applications" ,IEEE International Conference on Electro Information Technology (EIT), pp. 442-446, Lincoln, NE, USA, 2017.

[16] X. Liu, Z. Sheng, C. Yin, F. Ali, and D. Roggen, "Performance Analysis of Routing Protocol for Low Power and Lossy Networks (RPL) in Large Scale Networks", IEEE Internet of Things Journal, Vol. 4, No. 6. pp. 2172-2185, 2017.

[17] S. Kharche and S. Pawar, "Stability Model for RPL with Minimum Rank Hysterisis Objective Function in 6LoWPAN" , Int. J. Pure Appl. Math, Vol. 118, No. 5 Special Issue, pp. 731-737, 2018.

[18] S. A. Rafea and A. A. Kadhim, "Routing with Energy Threshold for WSN-IoT Based on RPL Protocol", IJCCCE Journal, Vol. 19, No. 1, pp. 71-81, 2019.

[19] D. Navani, S. Jain, and M. Singh, "The Internet of Things ( IoT ): A Study of Architectural Elements" , 13th Int. Conf. Signal-Image Technol. Internet-Based Syst. , pp. 473-478, 2017.

[20] S. Thombre, R. U. Islam, K. Andersson, and M. S. Hossain, "IP Based Wireless Sensor Networks: Performance Analysis Using Simulations and Experiments", JoWUA, Vol. 7, No. 3, pp. 53-76, 2016.

[21] A. Al-Fuqaha, M. Guizani, M. Mohammadi, M. Aledhari, and M. Ayyash, "Internet of Things: A survey on Enabling Technologies, Protocols, and Applications", IEEE Commun. Surv. Tutorials, IEEE Org. , Vol. 17, No. 4, pp. 2347-2376, 2015.

[22] K. Naito, "A Survey on the Internet of Things: Standards, Challenges and Future Prospects", J. Inf. Process, Vol. 25, No. 0, pp. $23-31,2017$.

[23] T. Salman and R. Jain, "Networking Protocols and Standards for Internet of Things" , Internet of Things and Data Analytics Handbook, pp. 215-238, 2017.

[24] A. Parasuram, D. Culler, and R. Katz, "An Analysis of The RPL Routing Standard for Low Power and Lossy Networks" , Technical Report, Electrical Engineering and Computer Sciences, University of California at Berkeley, pp. 1-98, 2016.

[25] O. Gnawali and P. Levis, "The Minimum Rank with Hysteresis Objective Function" , DOI 10.17487/RFC6719, Sept. , 2012.

[26] P. Thubert, "Objective Function Zero for the Routing Protocol for Low-Power and Lossy Networks (RPL) " , DOI 10.17487 / RFC6552, March, 2012.

[27] M. Kim and D. Barthel, "Routing Metrics Used for Path Calculation in Low Power and Lossy Networks" , pp. 1-30, 2011.

[28] H. Kim, J. Ko, D. Culler, and J. Paek, "Challenging The IPv6 Routing Protocol for Low-Power and Lossy Networks (RPL): A Survey" , IEEE Commun. Surv. Tutorials, Vol. 19, No. 4, 2017.

[29] Zolertia Zl, Datasheet, "Low Power Wireless Sensor Module" , http://www.ncs-in.com/downloads/Z1-RevC-Datasheet.pdf. 